W. Hemmer

C. Starck

\section{Pacemaker leads: special and surgical aspects}

The topic of pacemaker electrodes will be continued and concluded with this issue of Herzschrittmachertherapie und Elektrophysiologie. The manuscripts of issue 3/ 2001 dealt with the fundamentals of cardiac stimulation, the results of conventional pacemaker leads and the design and electrical characteristics of modern electrodes.

Contents of this issue are, first of all, special therapeutical concepts requiring specific characteristics of the leads used. B. Nowak presents the latest development in VDD stimulation, which became established as an alternative to DDD stimulation in patients with isolated AV-block. The second article of M. Anelli-Monti et al. summarizes today's possibilities of an automatic pacing threshold adjustment. In association with suitable electrodes, this concept

Prof. Dr. W. Hemmer (

Dr. C. Starck

Sana Herzchirurgische Klinik

Herdweg 2

70714 Stuttgart, Germany already functions extremely reliable and therefore will very possibly be part of future pacemaker systems. Resynchronization therapy of severe cardiac insufficiency with biventricular stimulation is nowadays a major topic in electrophysiology. Transvenous implantation of left ventricular leads via the coronary sinus requires special electrodes; the current development is presented in detail by J. Sperzel et al.

The second part of this issue addresses surgical aspects. Nowadays implantation of pacemaker electrodes is performed standardly using the cephalic vein as venous access, especially by surgeons. An alternative is the puncture of the subclavian vein and insertion of the leads using the Seldinger technique. D. Kronski and $H$. Haas show in a practically orientated article that considerable time savings and a low complication rate can be achieved with primary subclavian vein puncture in case of an experienced physician.

The last two articles deal with a negative aspect of pacemaker therapy: complications which require lead extraction. Continuous traction, which used to be popular but also problematic, was replaced by new, highly efficient percutaneous techniques. These techniques, including the latest developments as laser and electrosurgical dissection sheaths, are critically assessed by $U$. Wolfhard. We regard failure of percutaneous techniques or a high risk of embolization as an indication for surgical lead removal with or without the use of cardiopulmonary bypass. Our personal experience with surgical lead extraction on 16 patients is presented in another article.

The intention of these two issues was to provide the reader with a current summary on the topic of pacemaker electrodes. Also in the name of the editorial staff and the publisher, we would like to thank the authors once more, who without exception submitted their manuscripts in time, despite an enormous workload, and therefore are also responsible for the successful outcome of this project. 